\title{
An application of interacting shear flows theory: exact solution for unsteady oblique stagnation point flow
}

\author{
Guibo Li · Minguo Dai · Zhi Gao
}

Received: 14 February 2006 / Accepted: 29 May 2006 / Published online: 1 September 2006

(C) Springer-Verlag 2006

\begin{abstract}
An analytical solution of the governing equations of the interacting shear flows for unsteady oblique stagnation point flow is obtained. It has the same form as that of the exact solution obtained from the complete NS equations and physical analysis and relevant discussions are then presented.
\end{abstract}

Keywords Navier-Stokes equations · Interacting shear flows · Unsteady oblique stagnation point flow . Exact solution

\section{Introduction}

In Fluid Mechanics, researches on the exact solutions of NS equations are important for the following reasons [1, 2]: (1) the exact solutions represent fundamental fluid-dynamic flows. Because the exact solutions always have an analytical form, these fundamental flows can be studied in more detail. (2) The exact solutions can serve as standards for checking the accuracy of solutions obtained by other methods (e.g. numerical method, asymptotic method and empirical method etc.). Hence, the attempt at the exact solutions of the NS equations has never been interrupted from the very beginning $[1,3]$.

The English text was polished by Yunming Chen.

G. Li

College of Physical and Environmental Oceanography,

Ocean University of China, Qingdao 266003, China

e-mail: liguiboo@hotmail.com

G. Li (凶) · M. Dai · Z. Gao

LHD, Institute of Mechanics, Chinese Academy of Sciences, Beijing 100080, China
The two-dimensional stagnation point flow, the axisymmetric stagnation point flow and the three-dimensional oblique stagnation point flow are three examples of the interacting shear flows (ISF). The conception of the interacting shear flows and its fundamental governing equations were introduced by Gao Zhi $[3,4]$. For the cases of two-dimensional and axisymmetric stagnation point flows, the governing equations of ISF is consistent with the diffusion-parabolized Navier-Stokes (DPNS) equations, the analytical solution of the DPNS equations and the exact solution of the complete NS equations are the same as pointed out in Ref. [5]. In this paper, the unsteady three-dimensional oblique stagnation point flow is further studied. We come to the conclusion that the analytical solution of ISF's governing equations i.e. DPNS equations and the exact solution of the complete NS equations are the same as well. Physical analysis and some discussion are then presented.

\section{Unsteady three-dimensional oblique stagnation point flow - an example of interacting shear flows and its fundamental governing equations}

The interacting shear flows is composed of a thin viscous shear layer with a large length scale in the convection-dominant direction and a small length scale in the convection-diffusion competition direction and an outside inviscid flow which has nonlinear interaction with the thin viscous shear layer. For the case of unsteady three-dimensional oblique stagnation point flow studied in this paper, the thin viscous shear layer is at the neighborhood of the wall ( $x y$ plane), the $x$ - and $y$-directions are the convection-dominant directions, the 
convection-diffusion competition direction is the normal of the wall i.e. the $z$-direction. From the physical definition of convection-dominant and convection-diffusion competition [3, 4], we have

$u \frac{\partial f}{\partial x} \gg v \frac{\partial^{2} f}{\partial x^{2}}, \quad v \frac{\partial f}{\partial y} \gg v \frac{\partial^{2} f}{\partial y^{2}}$,

$w \frac{\partial f}{\partial z} \sim v \frac{\partial^{2} f}{\partial z^{2}}$,

where $f=u, v$ and $w . u, v$ and $w$ are the components of flow velocity in the $x$-, $y$ - and $z$-direction, respectively.

Using the continuity equation $\partial u / \partial x \sim \partial v / \partial y \sim$ $\partial w / \partial z$, and dropping the terms whose order of magnitude is smaller than the convection-diffusion competition terms (2), we obtain the following simplest governing equations of the unsteady three-dimensional oblique stagnation point flow which is also consistent with the DPNS equations $[3,4]$.

In a three-dimensional Cartesian coordinate, where the $z$-direction is the normal of the wall, the unsteady governing equations of oblique stagnation point flow have the following form.

$\frac{\partial u}{\partial x}+\frac{\partial v}{\partial y}+\frac{\partial w}{\partial z}=0$

$\frac{\partial u}{\partial t}+u \frac{\partial u}{\partial x}+v \frac{\partial u}{\partial y}+w \frac{\partial u}{\partial z}+\frac{1}{\rho} \frac{\partial p}{\partial x}=v \frac{\partial^{2} u}{\partial z^{2}}$,

$\frac{\partial v}{\partial t}+u \frac{\partial v}{\partial x}+v \frac{\partial v}{\partial y}+w \frac{\partial v}{\partial z}+\frac{1}{\rho} \frac{\partial p}{\partial y}=v \frac{\partial^{2} v}{\partial z^{2}}$,

$\frac{\partial w}{\partial t}+u \frac{\partial w}{\partial x}+v \frac{\partial w}{\partial y}+w \frac{\partial w}{\partial z}+\frac{1}{\rho} \frac{\partial p}{\partial z}=v \frac{\partial^{2} w}{\partial z^{2}}$.

From this we can draw a conclusion that in the case of Cartesian coordinate system the ISF's governing equations is consistent with the DPNS equations presented in late 1960s. For convenience and clarity we will still use the name DPNS equations below.

\section{Analytical solution of DPNS equations for unsteady three-dimensional oblique stagnation point flow}

For the two-dimensional stagnation point flow and the axisymmetric stagnation point flow, a nonrotational flow field has always been assumed. i.e. there exists potential function [6]. However, for the oblique stagnation point flow, we assume the flow field rotational and assume the outer flow varying as $(1-\beta t)^{-1}$ in order to make comparison with the existent exact solutions [7] of

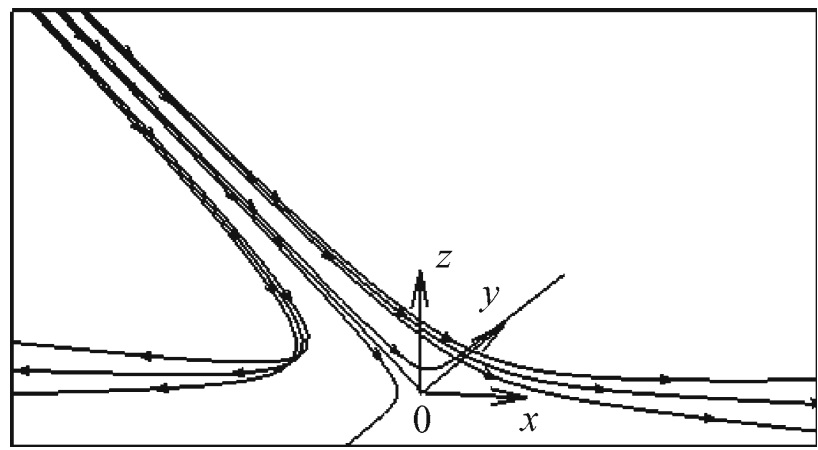

Fig. 1 Streamlines of the outside inviscid flow field ( 0 is the stagnation point)

NS equations.

$$
\begin{aligned}
U & =\frac{\alpha(x+\gamma z)}{1-\beta t}, \\
V & =\frac{\beta y}{1-\beta t}, \\
W & =-\frac{(\alpha+\beta) z}{1-\beta t}, \\
P & =\frac{-\rho}{(1-\beta t)^{2}}\left(\frac{\alpha(\alpha+\beta)}{2}\left(x^{2}+z^{2}\right)+\beta^{2} y^{2}+\text { const }\right),
\end{aligned}
$$

where $\alpha, \beta, \gamma$ are constants, $t$ is the time, $\rho$ the density and $P$ the pressure. The solutions (7) and (8) satisfy the nonviscous form of the governing equations (3)-(6), i.e. the Euler equations. Without loss of generality, we choose the $x$-axis so that $\alpha>0, \beta$ may be any positive or negative constant such that $\alpha+\beta>0$ in order to insure the $W$ component to be in the negative $z$-direction. Figure 1 shows the streamlines of the outside inviscid flow field at an inclination angle of $45^{\circ}$.

In the thin viscous shear layer at the neighborhood of the wall, the solution should satisfy the governing equations (3)-(6). We assume that the following viscous solution has a form similar to the one used in Ref. [7].

$$
\begin{aligned}
u= & \alpha\left(\frac{x f^{\prime}(\eta)}{1-\beta t}+\frac{\gamma g(\eta)}{\sqrt{1-\beta t}}\right), \\
v= & \frac{\beta y h^{\prime}(\eta)}{1-\beta t}, \\
w= & \frac{-(\alpha f(\eta)+\beta h(\eta))}{\sqrt{1-\beta t}}\left(\frac{v}{\beta}\right)^{1 / 2}, \\
p= & \frac{-\rho}{(1-\beta t)^{2}}\left(\frac{\alpha(\alpha+\beta)}{2} x^{2}+\frac{\alpha(\alpha+\beta)}{2}\right. \\
& \left.\times F(\eta) v(1-\beta t)+\beta^{2} y^{2}+\text { const }\right),
\end{aligned}
$$

where $\eta=\frac{z}{\sqrt{1-\beta t}}\left(\frac{\beta}{v}\right)^{1 / 2}, v$ is the kinematic viscosity, $-\infty<t \leq 1 / \beta$. 


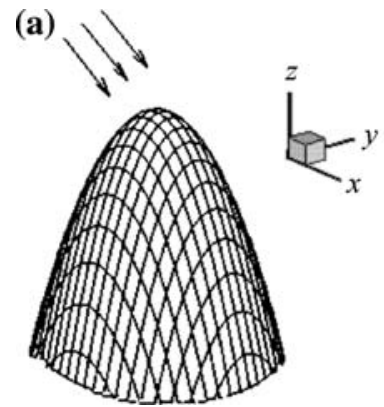

(b)

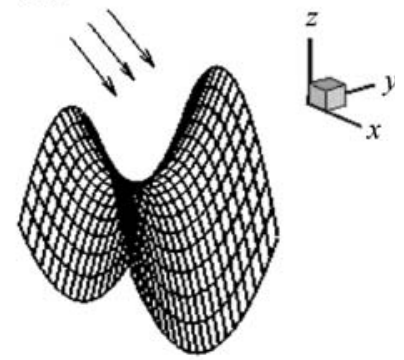

Fig. 2 Geometry and the coordinate system at the neighborhood of the stagnation point

On the $z=0$ plane, the no-slip condition is satisfied, and at infinity the solution (9) and (10) approaches the outer inviscid flow (7) and (8). The boundary conditions are

$z=0: \quad u=v=w=0$,

$z \rightarrow \infty: \quad u \rightarrow U, \quad v \rightarrow V$.

By substituting the solutions (9) and (10) into the DPNS equations (3)-(6), a set of nonlinear ordinary differential equations are obtained as follows

$$
\begin{aligned}
& \frac{A}{2} \eta f^{\prime \prime}+A f^{\prime}+f^{\prime} 2-(f+A h) f^{\prime \prime}=1+A+A f^{\prime \prime \prime} \\
& \frac{A}{2} \eta h^{\prime \prime}+A h^{\prime}+A h^{\prime} 2-(f+A h) h^{\prime \prime}=2 A+A h^{\prime \prime \prime}
\end{aligned}
$$

the boundary conditions are

$f(0)=f^{\prime}(0)=h(0)=h^{\prime}(0)=g(0)=F(0)=0$,

$f^{\prime}(\infty)=h^{\prime}(\infty)=g^{\prime}(\infty)=1$,

where the parameter $A=\frac{\beta}{\alpha}$.

According to Ref. [6], the parameter $A$ is determined by the velocity components $U$ and $V$ of the outer inviscid flow and characterizes the type of curvature of the surface around the stagnation point. Considering $\alpha>0$, the case $A>0, \beta>0$ corresponds to the accelerating flow and the nodal point of attachment shown in Fig. 2a, while $A<0, \beta<0$ corresponds to the decelerating flow and the saddle point of attachment shown in Fig. $2 b$.

Now, we can find that the analytical solution of DPNS equations and the exact solution of the complete NS equations have the same form. The complete NS equations read

$$
\begin{aligned}
\frac{\partial u}{\partial t} & +u \frac{\partial u}{\partial x}+v \frac{\partial u}{\partial y}+w \frac{\partial u}{\partial z}+\frac{1}{\rho} \frac{\partial p}{\partial x} \\
& =v\left(\frac{\partial^{2} u}{\partial x^{2}}+\frac{\partial^{2} u}{\partial y^{2}}+\frac{\partial^{2} u}{\partial z^{2}}\right), \\
\frac{\partial v}{\partial t} & +u \frac{\partial v}{\partial x}+v \frac{\partial v}{\partial y}+w \frac{\partial v}{\partial z}+\frac{1}{\rho} \frac{\partial p}{\partial y} \\
& =v\left(\frac{\partial^{2} v}{\partial x^{2}}+\frac{\partial^{2} v}{\partial y^{2}}+\frac{\partial^{2} v}{\partial z^{2}}\right), \\
\frac{\partial w}{\partial t} & +u \frac{\partial w}{\partial x}+v \frac{\partial w}{\partial y}+w \frac{\partial w}{\partial z}+\frac{1}{\rho} \frac{\partial p}{\partial z} \\
& =v\left(\frac{\partial^{2} w}{\partial x^{2}}+\frac{\partial^{2} w}{\partial y^{2}}+\frac{\partial^{2} w}{\partial z^{2}}\right) .
\end{aligned}
$$

The details of the flow field can be obtained by solving the ordinary differential equations (13)-(18). We can solve Eq. (13)-(18) numerically as follows. Equations (13)-(15) are firstly integrated as an initial problem by using shooting method. After $f(\eta), f^{\prime}(\eta), h(\eta)$ and $h^{\prime}(\eta)$ are known, Eq. (16) can be integrated and $F(\eta)$ be obtained by referring to the initial condition $F(0)=0$. At last, the pressure change across the boundary layer can be calculated from Eq. (10).

\section{Result analyses}

As mentioned above, the parameter $A$ may be any positive or negative constant. As a matter of fact, the range of $A$ from -1 to 1 effectively covers most of the flows. In what follows $A$ is limited to the range from 0 to 1 .

For the sake of clarity and convenience, we normalize all lengths, velocities and time by $\sqrt{\nu / \beta}, \sqrt{\nu \beta}$ and $1 / \beta$

$$
\begin{aligned}
\bar{u} & =\frac{1}{A}\left(\frac{\bar{x} f^{\prime}(\eta)}{1-\bar{t}}+\frac{\bar{\gamma} g(\eta)}{\sqrt{1-\bar{t}}}\right), \\
\bar{v} & =\frac{\bar{y} h^{\prime}(\eta)}{1-\bar{t}}, \\
\bar{w} & =\frac{-(f(\eta) / A+h(\eta))}{\sqrt{1-\bar{t}}},
\end{aligned}
$$

where, $\eta=\frac{\bar{z}}{\sqrt{1-\bar{t}}}, \bar{\gamma}=\gamma\left(\frac{\beta}{\nu}\right)^{1 / 2}$.

As a result, the shear stresses on the $z=0$ plane are

$$
\begin{aligned}
& \bar{\tau}_{z x}=\left.\mu \frac{\partial \bar{u}}{\partial z}\right|_{0}=\frac{\mu}{A}\left(\frac{\bar{x} f^{\prime \prime}(0)}{(1-\bar{t})^{3 / 2}}+\frac{\bar{\gamma} g^{\prime}(0)}{1-\bar{t}}\right), \\
& \bar{\tau}_{z y}=\left.\mu \frac{\partial \bar{v}}{\partial z}\right|_{0}=\mu \frac{\bar{y} h^{\prime \prime}(0)}{(1-\bar{t})^{3 / 2}} .
\end{aligned}
$$

Equations (13)-(15) are solved simultaneously by the fifth-order Runge-Kutta-Fehlberg algorithm. Table 1 


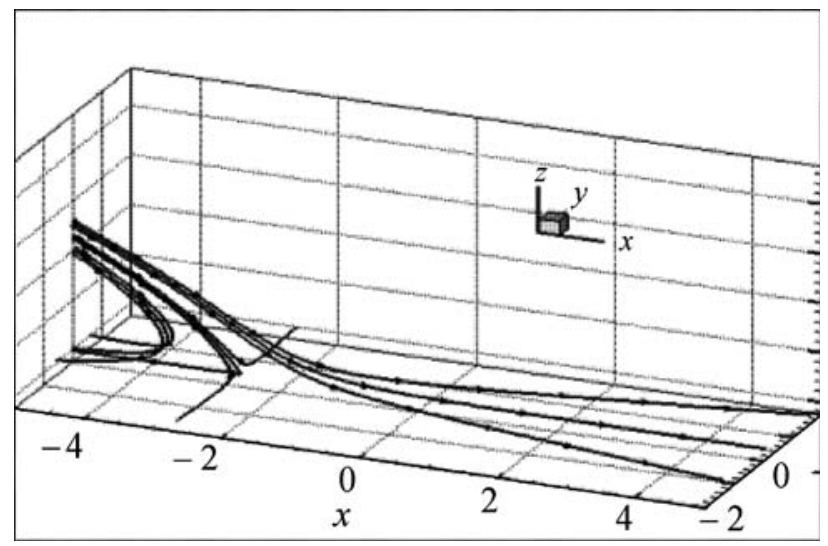

Fig. 3 Streamlines of $\bar{y}=0, \bar{t}=-2, \theta=30^{\circ}$

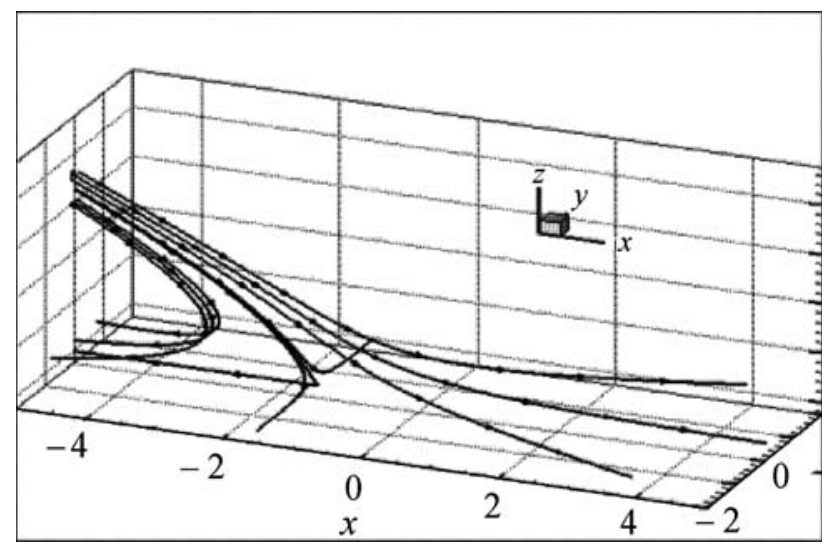

Fig. 4 Streamlines of $\bar{y}=0, \bar{t}=-2, \theta=45^{\circ}$

gives the resulting values of $f^{\prime}(\eta), h^{\prime}(\eta)$ and $g(\eta)$ for $A=0.25,0.5,0.75$ and 1 , respectively. Table 2 gives the resulting values of $f^{\prime \prime}(0), h^{\prime \prime}(0)$ and $g^{\prime}(0)$ for $A=0.25$, $0.5,0.75$ and 1 , respectively. For the case of $A=0$, it is reduced to the case of two-dimensional flow.

From Table 1 we can find the relation that $f=h$ when $A=1$, i.e. $\alpha=\beta>0$. The corresponding flow is the circular impinging jet.

The stagnation point flow is

$(\bar{x}, \bar{y}, \bar{z})=\left(-\frac{\bar{\gamma} g^{\prime}(0)}{f^{\prime \prime}(0)} \sqrt{1-\bar{t}}, 0,0\right)$,

while $(\bar{x}, \bar{y}, \bar{z})=(0,0,0)$ represents the inviscid outer flow. Because of viscous effects, the stagnation point moves to the upstream side at a distance of $\frac{\bar{\gamma} g^{\prime}(0)}{f^{\prime \prime}(0)} \sqrt{1-\bar{t}}$. As time $\bar{t} \rightarrow 1$, the stagnation point moves toward the origin $(0,0,0)$. In order to explain the relation between the position of the stagnation point and time and the inclination angle of the outer inviscid flow field, Figs. 3, 4,5 and 6 show the streamlines for time equal to -2 and 0 when the inclination angle of the impinging jet are $30^{\circ}$ and $45^{\circ}$, respectively.

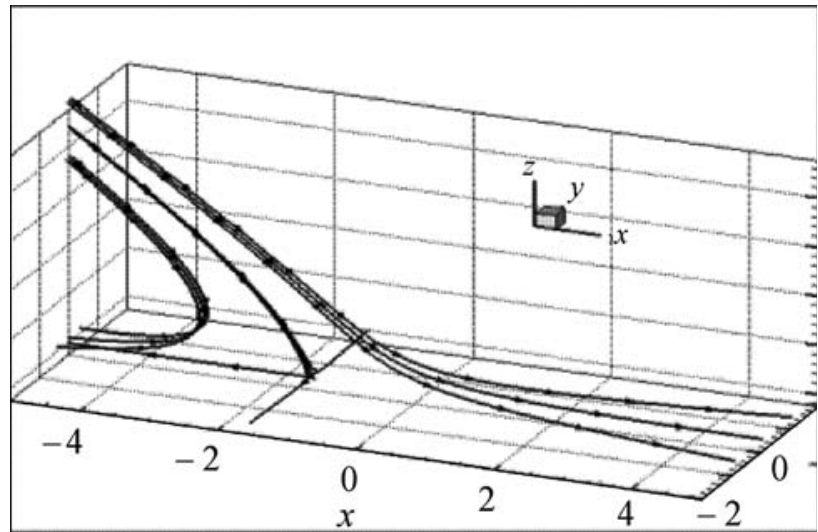

Fig. 5 Streamlines of $\bar{y}=0, \bar{t}=0, \theta=30^{\circ}$

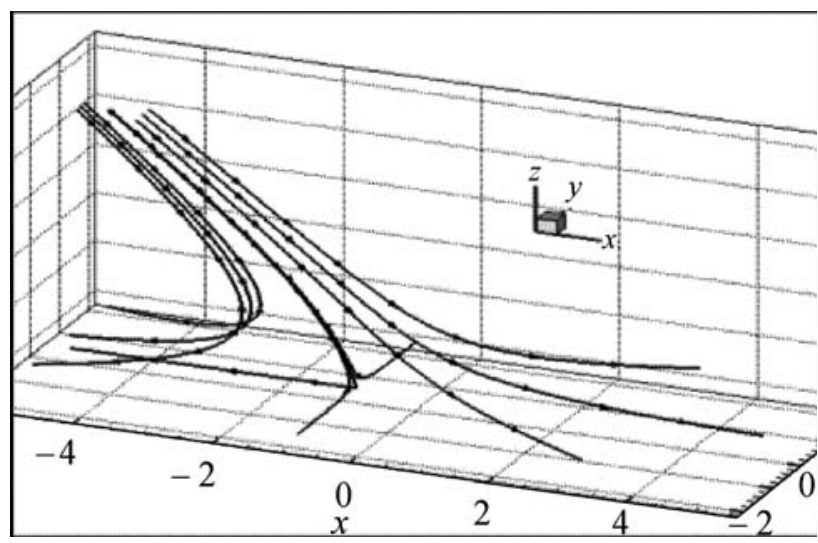

Fig. 6 Streamlines of $\bar{y}=0, \bar{t}=0, \theta=45^{\circ}$

When the inclination angle keeps unchanged, the stagnation point moves toward the origin as the time increases. The stagnation point approaches the origin as the time approaches 1 . On the other hand, when the time keeps unchanged, the stagnation point moves toward the origin as the inclination angle increases. The stagnation point coincides with the origin when the inclination angle is equal to $90^{\circ}$.

\section{Conclusions}

In this paper, we proved that the three-dimensional oblique stagnation point flow is an example of the interacting shear flow, and that the analytical solution of the DPNS equations for this flow has the same form as that of the exact solution obtained from the complete NS equations. Since many natural flows are interacting shear flows, further studies on the interacting shear flows and the DPNS equations can offer us a better understanding of certain natural phenomena and their mechanism by comparing them with the exact solution 
Table 1 Resulting values of $f^{\prime}(n), h^{\prime}(n)$, and $g(\eta)$ for $\mathrm{A}=0.25,0.5,0.75$ and 1 , respectively

\begin{tabular}{|c|c|c|c|c|c|c|c|}
\hline$\eta$ & $f^{\prime}$ & $h^{\prime}$ & $g$ & $\eta$ & $f^{\prime}$ & $h^{\prime}$ & $g$ \\
\hline \multicolumn{4}{|c|}{$A=0.25$} & \multicolumn{4}{|c|}{$A=0.5$} \\
\hline 0 & 0 & 0 & 0 & 0 & 0 & 0 & 0 \\
\hline 0.1028 & 0.1121 & 0.1451 & 0.0401 & 0.1019 & 0.1249 & 0.1453 & 0.0417 \\
\hline 0.2078 & 0.2145 & 0.2739 & 0.0814 & 0.2059 & 0.2382 & 0.2746 & 0.0846 \\
\hline 0.3213 & 0.3134 & 0.3936 & 0.127 & 0.3175 & 0.3458 & 0.3943 & 0.1317 \\
\hline 0.4432 & 0.4075 & 0.5026 & 0.1775 & 0.4368 & 0.4465 & 0.5033 & 0.1835 \\
\hline 0.5732 & 0.4956 & 0.5994 & 0.2336 & 0.5641 & 0.5395 & 0.6005 & 0.2413 \\
\hline 0.6421 & 0.5377 & 0.6436 & 0.2644 & 0.6316 & 0.5834 & 0.6452 & 0.2731 \\
\hline 0.78 & 0.6133 & 0.7194 & 0.3289 & 0.7668 & 0.6611 & 0.7218 & 0.3397 \\
\hline 0.8576 & 0.6512 & 0.7555 & 0.3669 & 0.8423 & 0.6991 & 0.7581 & 0.3788 \\
\hline 0.9352 & 0.6859 & 0.7873 & 0.4062 & 0.9178 & 0.7336 & 0.7903 & 0.4193 \\
\hline 1.2366 & 0.7954 & 0.879 & 0.5729 & 1.0689 & 0.7929 & 0.8437 & 0.505 \\
\hline 1.5213 & 0.8679 & 0.9313 & 0.7521 & 1.5495 & 0.9145 & 0.9435 & 0.8215 \\
\hline 2.0458 & 0.9463 & 0.9779 & 1.1379 & 2.0354 & 0.9698 & 0.9824 & 1.2074 \\
\hline 2.4947 & 0.9777 & 0.9924 & 1.5172 & 2.5119 & 0.9907 & 0.9952 & 1.635 \\
\hline 3.1344 & 0.9947 & 0.9986 & 2.1106 & 3.0317 & 0.9979 & 0.999 & 2.1336 \\
\hline 3.527 & 0.998 & 0.9996 & 2.4922 & 3.5311 & 0.9996 & 0.9998 & 2.6268 \\
\hline 4.1221 & 0.9996 & 0.9999 & 3.0813 & 4.0055 & 0.9999 & 1 & 3.0997 \\
\hline 4.4958 & 0.9999 & 1.0000 & 3.4539 & 4.5678 & 1 & 1 & 3.6617 \\
\hline \multicolumn{4}{|c|}{$A=0.75$} & \multicolumn{4}{|c|}{$A=1.0$} \\
\hline 0 & 0 & 0 & 0 & 0 & 0 & 0 & 0 \\
\hline 0.1009 & 0.136 & 0.1455 & 0.043 & 0.0998 & 0.1457 & 0.1457 & 0.044 \\
\hline 0.2038 & 0.2585 & 0.2753 & 0.0873 & 0.2015 & 0.2761 & 0.2761 & 0.0893 \\
\hline 0.3135 & 0.373 & 0.3952 & 0.1354 & 0.3093 & 0.3962 & 0.3962 & 0.1383 \\
\hline 0.43 & 0.4784 & 0.504 & 0.1883 & 0.423 & 0.5049 & 0.5049 & 0.1917 \\
\hline 0.5544 & 0.5745 & 0.6018 & 0.2472 & 0.5445 & 0.6031 & 0.6031 & 0.2514 \\
\hline 0.6205 & 0.6195 & 0.6469 & 0.2798 & 0.6091 & 0.6486 & 0.6486 & 0.2844 \\
\hline 0.7527 & 0.6979 & 0.7244 & 0.348 & 0.7383 & 0.727 & 0.727 & 0.3538 \\
\hline 0.8261 & 0.7355 & 0.7609 & 0.3879 & 0.8097 & 0.7638 & 0.7638 & 0.3942 \\
\hline 0.9731 & 0.7992 & 0.822 & 0.4723 & 0.9526 & 0.8253 & 0.8253 & 0.4797 \\
\hline 1.1147 & 0.8482 & 0.8679 & 0.5599 & 1.024 & 0.8507 & 0.8507 & 0.525 \\
\hline 1.5083 & 0.9354 & 0.9465 & 0.837 & 1.5285 & 0.9569 & 0.9569 & 0.8943 \\
\hline 2.0189 & 0.9823 & 0.9862 & 1.2631 & 2.0491 & 0.9908 & 0.9908 & 1.3518 \\
\hline 2.5168 & 0.996 & 0.9971 & 1.7277 & 2.5147 & 0.9982 & 0.9982 & 1.7986 \\
\hline 3.0627 & 0.9994 & 0.9996 & 2.2628 & 3.0304 & 0.9998 & 0.9998 & 2.3092 \\
\hline 3.5426 & 0.9999 & 0.9999 & 2.7407 & 3.5034 & 1 & 1 & 2.7814 \\
\hline 4.0235 & 1 & 1 & 3.2212 & 4.0902 & 1 & 1 & 3.3681 \\
\hline 4.5 & 1 & 1 & 3.6977 & 4.5131 & 1 & 1 & 3.791 \\
\hline
\end{tabular}

Table 2 Resulting values of $f^{\prime \prime}(0), h^{\prime \prime}(0)$ and $g^{\prime}(0)$ for $\mathrm{A}=0.25,0.5,0.75$ and 1 , respectively

\begin{tabular}{lllll}
\hline & $A=0.25$ & $A=0.5$ & $A=0.75$ & $A=1.0$ \\
\hline$f^{\prime \prime}(0)$ & 1.1511 & 1.2991 & 1.4334 & 1.5570 \\
$h^{\prime \prime}(0)$ & 1.5102 & 1.5242 & 1.5400 & 1.5570 \\
$g^{\prime}(0)$ & 0.3891 & 0.4082 & 0.4253 & 0.4401 \\
\hline
\end{tabular}

of NS equations and the solution of boundary layer equations. Significance of the study on the exact solutions of DPNS equations is revealed more clearly by the fact that any high Reynolds number flow consists of three basic flows, that is the flow with convection-dominant in all spatial directions, the interacting shear flow (i.e. flow with convection-dominant in part direction and convection-diffusion competition in part direction) and flow with convection-diffusion competition in all directions, and that the NS flows are included in the thin viscous shear layer of the interacting shear flows. Since a number of exact solutions of Euler equations have been found in the past, the combination of these exact solutions of Euler equations and the exact solutions of the fundamental governing equations of interacting shear flows - DPNS equations will certainly offer an effective approach in searching for exact solutions of viscous flows. Finally, verification and validation of the precision in Computational Fluid Dynamics (CFD) is one of the most important problems [8]. Since the DPNS 
equations have been extensively used in CFD, the exact solutions of DPNS equations, like the exact solutions of NS equations, are rather helpful to CFD verification and validation.

\section{References}

1. Schlichting H., Gersten, K.: Boundary Layer Theory, 8th edn. Springer, Berlin Heidelberg New York (2000)

2. Wang, C.Y.: Exact solutions of the steady-state Navier-Stokes equations. Annu. Rev. Fluid Mech. 23, 159-177 (1991)

3. Gao, Z.: Vicous-inviscid interaction flow theory (in Chinese). Acta Mech. Sin. 22(1), 9-19 (1990)
4. Gao, Z.: Significance and use of basic equation system governing high Reynolds ( $R e$ ) number flows and diffusionparabolized Navier-Stokes (DPNS) equations (in Chinese). Adv. Mech. 35(3), 427-438 (2005)

5. Gao, Z.: Some exact solutions of the simplified Navier-Stokes equations (in Chinese). Acta Mech. Sin. 17(3), 201-210 (1985)

6. Cheng, E.H., Ozisik, M.N., Williams, J.C. III: Nonsteady three-dimensional stagnation-point flow. J. Appl. Mech. 38(1), 282-287 (1971)

7. Wang, C.Y.: The unsteady oblique stagnation point flow. Phys. Fluids 28(7), 2046-2049 (1985)

8. Oberkampf, W.L., Trucan, T.G.: Verification and validation in computational fluid dynamics. SAND 2002-0529, 2002 